\title{
Relationship between Dual-Energy X-Ray Absorptiometry-Derived Appendicular Lean Tissue Mass and Total Body Skeletal Muscle Mass Estimated by Ultrasound
}

\author{
Takashi Abe ${ }^{1,2^{*}}$, Nicole C. Dabbs ${ }^{1}$, Vinayak K. Nahar ${ }^{1}$, M. Allison Ford ${ }^{1}$, Martha A. Bass ${ }^{1}$, \\ Mark Loftin ${ }^{1}$ \\ ${ }^{1}$ Department of Health, Exercise Science, \& Recreation Management, School of Applied Science, The University of Mississippi, \\ Oxford, USA; ${ }^{2}$ Department of Kinesiology, School of Public Health, Indiana University, Bloomington, USA. \\ Email: *t12abe@gmail.com
}

Received April 12 $2^{\text {th }}, 2013$; revised May $15^{\text {th }}, 2013$; accepted May $23^{\text {rd }}, 2013$

Copyright (C) 2013 Takashi Abe et al. This is an open access article distributed under the Creative Commons Attribution License, which permits unrestricted use, distribution, and reproduction in any medium, provided the original work is properly cited.

\begin{abstract}
Dual-energy X-ray absorptiometry (DXA) is an attractive method for evaluating sarcopenia, age-related loss of skeletal muscle mass and function, using appendicular lean tissue (aLT) mass for criteria of diagnosis, although minimal radiation is exposed. Skeletal muscle (SM) mass can be estimated by using ultrasound-measured muscle thickness (MTH). However, the association between these two methods is unclear. To examine the relationship between DXA-derived aLT mass and total body SM mass estimated by ultrasound, thirty-six healthy adults (18 men and 18 women) aged 19 65 years participated in this study. Ultrasound-measured muscle thickness was used to estimate the total SM mass. DXA was used to estimate whole body and regional body composition, and aLT mass was considered equivalent to the sum of lean tissue in both the right and left arms and legs. Total SM mass $(26.3 \pm 4.4 \mathrm{~kg}$ for men and $15.7 \pm 2.6 \mathrm{~kg}$ for women) estimated by ultrasound was similar to DXA-estimated aLT mass $(24.5 \pm 3.8 \mathrm{~kg}$ for men and $15.7 \pm 2.7 \mathrm{~kg}$ for women). There was a strong correlation between DXA-measured aLT mass and total SM mass estimated by ultrasound in men $(r=0.927, n=18)$ and women $(r=0.931, n=18)$ as well as overall sample $(r=0.975, n=36)$. The ratio of total SM mass to aLT mass was 1.07 for men and 1.00 for women. These results suggest that DXA-derived aLT mass can be accurately predicted from ultrasound estimated total SM mass, although the predicted value may underestimate in men (approximately $7 \%$ at the group level).
\end{abstract}

Keywords: Body Composition; Skeletal Muscle Mass; Sarcopenia; B-Mode Ultrasonography

\section{Introduction}

Magnetic resonance imaging (MRI) and computed tomography (CT) are gold standards for estimating total and regional skeletal muscle (SM) mass in research. Dualenergy X-ray absorptiometry (DXA) is a preferred alternative method for estimating body composition, especially bone mineral density and appendicular lean tissue (aLT) mass measurements. The DXA-derived aLT mass as a major criteria of diagnosis for age-related sarcopenia is previously reported in many studies [1,2]. However, these techniques are costly and access to the equipment is limited. In addition, these equipments are not portable, which may preclude the measurements in large samples ${ }^{*}$ Corresponding author. of epidemiological studies.

Bioimpedance analysis (BIA) is a portable alternative technique to estimate body composition such as body fat and lean body mass. Under standard conditions, BIA provides valid estimates of SM mass in healthy adults varying in age [3]. B-mode ultrasound is another technique employed to estimate SM mass. This method is very adaptable for field use. A previous study [4] reported a strong correlation between MRI-measured and ultrasound-predicted total SM mass in healthy adult men and women $(r=0.97)$, and the adult ultrasound-derived prediction equations were useful for estimating total SM mass for adolescents [5]. Because DXA provides a measure of aLT mass, Kim et al. [6,7] investigated the 
relationship between MRI-measured total SM mass and DXA-derived aLT mass in healthy adults and children. However, it is unknown whether there is a strong correlation between ultrasound predicted total SM mass and DXA-derived aLT mass. In the present study, we examined the relationship between aLT mass by DXA and total SM mass by ultrasound.

\section{Methods}

\subsection{Study Sample}

Participants were healthy adult men $(n=18)$ and women $(\mathrm{n}=18)$ who varied in age $(19-65 \mathrm{yr})$, and body mass index $\left(17.5-35.5 \mathrm{~kg} / \mathrm{m}^{2}\right)$. Participants were recruited from students and employees at the university through posted flyers and recruitment emails. University Institutional Review Board approved informed consent was obtained from all participants.

\subsection{Ultrasound-Predicted Total Skeletal Muscle Mass}

Total SM was estimated from ultrasound-derived prediction equation (sum of total nine sites) that converted muscle thickness (MTH) to SM [4]. A strong correlation $\left(\mathrm{R}^{2}=0.94\right)$ has previously been observed between magnetic resonance imaging-measured total SM and ultrasound-predicted total SM [4]. MTH was measured using B-mode ultrasound (Aloka SSD-500, Tokyo, Japan) at nine sites on the anterior and posterior aspects of the body (forearm, upper arm, trunk, thigh and lower leg) as previously described [8]. The measurements were taken while the subjects stood with their elbows and knees extended and relaxed. A 5-MHz scanning head was placed on the measurement site without depressing the dermal surface. Distortion of tissue due to excess compression was eliminated by observing that no movement of tissue occurred in the real-time ultrasound image. The subcutaneous adipose tissue-muscle interface and the musclebone interface were identified from the ultrasonic image, and the distance between two interfaces was recorded as MTH. The test-retest reliability for this method was less than $2 \%$, as described previously $[8,9]$.

\subsection{DXA-Estimated Appendicular Lean Tissue Mass}

Appendicular lean tissue (aLT) mass was estimated by DXA (Hologic QDR 4500 Discovery, Hologic Inc., Bedford, Mass., USA). The DXA system provided the mass of lean tissue, fat, and bone mineral for both the whole body and specific regions. After isolation from the trunk and head by use of specific anatomic landmarks, the aLT mass was considered equivalent to the total of lean tissue in both the right and left arms and legs.

\subsection{Statistical Analysis}

Results are expressed as means and standard deviation (SD). The difference between DXA and ultrasound and between men and women was tested for significance by using Student's $t$-test. Pearson product correlation was performed to determine the relationship between DXAestimated aLT mass and ultrasound-predicted total SM mass. A Bland-Altman plot [10] was used to evaluate the sensitivity of the relationship two variables estimated by DXA and ultrasound. p-values $<0.05$ were considered statistically significant.

\section{Results}

Ultrasound-predicted total SM mass was similar to DXAestimated aLT mass in men and women (Table 1). The ratio of total SM mass to aLT mass (SM:aLT ratio) was higher $(\mathrm{p}<0.05)$ in men $(1.07)$ than in women $(1.00)$. There was a strong correlation between the aLT mass and total SM mass in men $(\mathrm{r}=0.927, \mathrm{n}=18)$ and women $(\mathrm{r}=$ $0.931, \mathrm{n}=18)$ as well as overall sample $(\mathrm{r}=0.975, \mathrm{n}=$ 36). A regression expression between aLT mass and total SM mass was:

Total SM mass $(\mathrm{kg})=1.13 \times$ aLT mass $(\mathrm{kg})-1.83$ $(\mathrm{SEE}=1.46 \mathrm{~kg} ; \mathrm{n}=36)($ Figure 1).

aLT mass $(\mathrm{kg})=0.838 \times$ Total SM mass $(\mathrm{kg})+2.54$ $(\mathrm{SEE}=1.26 \mathrm{~kg} ; \mathrm{n}=36)$.

Bland-Altman plot revealed that there was no systematic bias between the difference and mean of two variables in men $(r=0.354, p=0.149)$ and women $(r=$

Table 1. Body composition and skeletal muscle mass in men and women.

\begin{tabular}{ccc}
\hline & Men $(\mathrm{n}=18)$ & Women $(\mathrm{n}=18)$ \\
\hline Age $(\mathrm{yr})$ & $40 \pm 18(21-65)$ & $33 \pm 13(19-62)$ \\
Body mass $(\mathrm{kg})$ & $77.4 \pm 10.5$ & $62.8 \pm 12.3$ \\
& $(61.4-90.9)^{\dagger}$ & $(43.6-90.8)$ \\
Height $(\mathrm{m})$ & $1.74 \pm 0.07$ & $1.61 \pm 0.04$ \\
& $(1.61-1.86)^{\dagger}$ & $(1.53-1.68)$ \\
BMI $\left(\mathrm{kg} / \mathrm{m}^{2}\right)$ & $25.5 \pm 2.3$ & $24.3 \pm 4.7$ \\
& $(22.2-29.3)$ & $(17.5-35.5)$ \\
Fat $(\%)$ & $24.1 \pm 5.2$ & $34.9 \pm 8.5$ \\
& $(11.8-32.1)$ & $(21.9-47.9)$ \\
aLT mass $(\mathrm{kg})$ & $24.5 \pm 3.8$ & $15.7 \pm 2.7$ \\
& $(18.3-32.5)^{\dagger}$ & $(12.1-22.5)$ \\
Total SM mass $(\mathrm{kg})$ & $26.3 \pm 4.4$ & $15.7 \pm 2.6$ \\
& $(17.6-33.6)^{\dagger}$ & $(11.0-20.5)$ \\
SM:aLT ratio & $1.07 \pm 0.07$ & $1.00 \pm 0.06$ \\
& $(0.97-1.17)^{*}$ & $(0.91-1.14)$ \\
\hline
\end{tabular}

Mean \pm SD; range in parentheses. aLT, appendicular lean tissue; SM, skeletal muscle; SM:aLT ratio, ratio of total SM mass to aLT mass; ${ }^{*} \mathrm{p}<0.05,{ }^{\dagger} \mathrm{p}$ $<0.01$, men vs. women. 


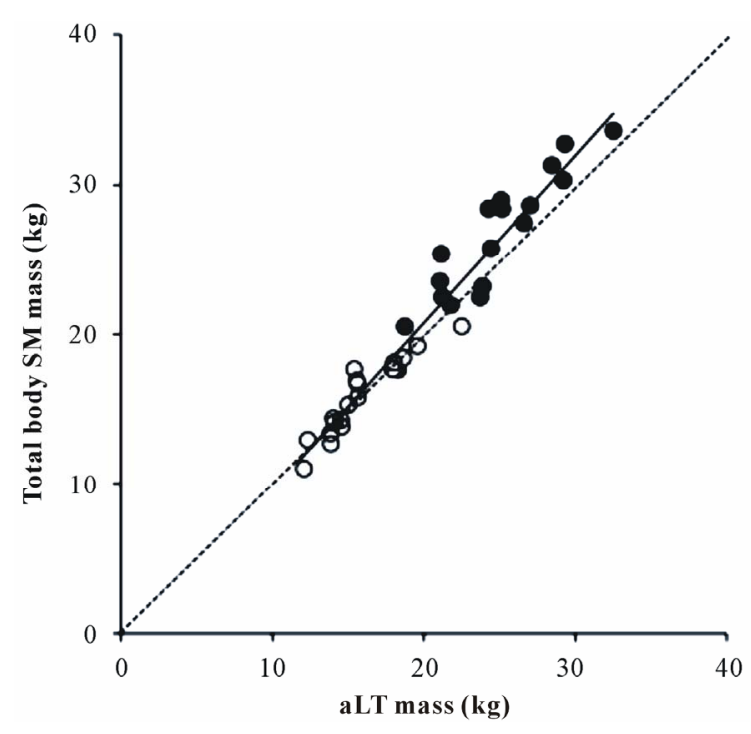

Figure 1. Relationship between total body skeletal muscle (SM) mass predicted by ultrasound and appendicular lean tissue (aLT) mass estimated by dual-energy $X$-ray absorptiometry in men $(\bullet)$ and women $(\circ)$. aLT mass $=0.838 \times$ total $\mathrm{SM}$ mass $+2.54,\left(\mathrm{R}^{2}=\mathbf{0 . 9 5 0}, \mathrm{n}=36\right)$.

$-0.085, p=0.736)$. Using overall sample, however, the difference was related to the mean of aLT mass and total SM mass $(r=0.560, p<0.001)$.

\section{Discussion}

The findings of the present study demonstrated that DXAestimated aLT mass was strongly related to ultrasoundpredicted total SM mass. In addition, there was no significant difference between the aLT mass and total SM mass in men and women.

It is known that a large proportion of SM mass is observed in the arms and legs including shoulders and glutei [11], and DXA-estimated lean tissue mass involves skin and connective tissue in the extremities [6]. On the other hand, ultrasound-derived prediction equations are a valid method to predict SM mass and an alternative to MRI measurement in healthy adults [4]. Using this equation, the predicted total SM mass includes the trunk SM mass, but not the skin and a large-scale of connective tissues in the extremities. These methodological differences between two techniques may explain the results that appendicular lean mass by DXA is similar to total body SM mass predicted by ultrasound.

Our findings showed that there was no difference between the aLT mass and total SM mass in healthy adults men and women, suggesting that a criterion for diagnosis of sarcopenia may be similar between two techniques. Although the exact definition of sarcopenia is still elusive, Baumgartner et al. [1] proposed the definition of sarcopenia such that severe sarcopenia is a DXA-esti- mated aLT mass index (aLT mass/height ${ }^{2}, \mathrm{~kg} / \mathrm{m}^{2}$ ) value of 2 standard deviations (SD) below the mean for young adults with a reference value in men of $7.26 \mathrm{~kg} / \mathrm{m}^{2}$. On the other hand, the reference value for severe sarcopenia (2 SD below) using ultrasound estimated SM mass index of $6.8 \mathrm{~kg} / \mathrm{m}^{2}$ in men [12]. The difference in the reference value between two techniques may include the influence of ethnicity (Americans vs. Japanese). However, it is unclear whether the severe sarcopenia is diagnosed with the same individuals in both criteria of the aLT mass index and total SM mass index. Further analyses are needed to be performed using a large sample of older adults.

A previous study [6] reported a simple regression model between MRI-measured total SM mass and DXAestimated aLT mass (Total SM mass $(\mathrm{kg})=1.19 \times \mathrm{aLT}$ mass $(\mathrm{kg})-1.01 ; \mathrm{R}^{2}=0.96$, SEE $\left.=1.63 \mathrm{~kg}\right)$. The $\mathrm{SM}$ : aLT ratio is almost same between men (1.16) and women (1.13). In the present study, our regression model is identical (Total SM mass $(\mathrm{kg})=1.13 \times \mathrm{aLT}$ mass $(\mathrm{kg})-1.83$; $\mathrm{R}^{2}=0.95$, SEE $=1.46 \mathrm{~kg}$ ) with the previous study [6]. However, the SM:aLT ratio was higher in men (1.07) than in women (1.00), which may relate to systematic bias between the difference and mean of aLT mass and total SM mass when overall sample was used. In addition, smaller SM:aLT ratios were found in the present study compared with the previous study [6]. A possible reason for the difference in SM:aLT ratio would be higher DXA-estimated aLT mass in the present study. Because DXA-estimated aLT mass is composed of muscle, connective tissue, skin, and the lean portion of adipose tissue, a larger adipose tissue may contributes to higher aLT mass. However, the differences in total body fat mass between the present and the previous study are relatively small in men (16 kg vs. $19 \mathrm{~kg})$ and women $(21 \mathrm{~kg}$ vs. 22 $\mathrm{kg}$ ). Furthermore, there is no significant correlation between total body fat mass and the difference of total SM mass and aLT mass in the present study $(\mathrm{r}=0.053, \mathrm{p}=$ 0.758).

Another possibility for the difference in SM:aLT ratio may be the distribution of muscle mass (arms, legs, and trunk) in the body. In the present study, 8 of 18 men and 2 of 18 women were performing sports activity (cycling and running). The SM:aLT ratio was 1.07 in sports active men $(\mathrm{n}=8)$ and 1.07 in other sedentary men $(\mathrm{n}=10)$. These results suggest that physical active levels may not be associated with lower percentage of the muscle mass distributions in the extremities. Thus, the smaller SM:aLT ratio seen in the present study is not supported by the reasons described above.

In the present study, we used an ultrasound technique to determine skeletal muscle size. Ultrasound measurement is simple, low cost, and easily applied in clinical assessment and field surveys with no health hazards. 
However, it is not possible to measure muscle size without intermuscular adipose tissue by ultrasound. Intermuscular adipose tissue is increased by advancement in age [13], and the accumulation of intermuscular adipose tissue may be a factor contributing to changing muscle size. Furthermore, because participants in this study were healthy adults men and women, it is uncertain if the results pertain to children and adolescents as well. Additional research into these issues is needed.

In conclusion, our results suggest that DXA-derived aLT mass can be accurately predicted from ultrasound estimated total SM mass, although the predicted value may overestimate in men (approximately 7\% at the group level).

\section{Acknowledgements}

The authors thank the individuals who participated in this study. None of the authors had financial or personal conflict of interest with regard to this study.

\section{REFERENCES}

[1] R. N. Baumgartner, K. M. Koehler, D. Gallagher, L. Romero, S. B. Heymsfield, R. Ross, P. J. Garry and R. D. Linderman, "Epidemiology of Sarcopenia among the Elderly in New Mexico," American Journal of Epidemiology, Vol. 147, No. 8, 1998, pp. 755-763. doi:10.1093/oxfordjournals.aje.a009520

[2] A. Y. Bijlsma, C. G. Meskers, C. H. Ling, M. Narici, S. E. Kurrle, I. D. Cameron, R. G. Westendrorp and A. B. Maier, "Defining Sarcopenia: The Impact of Different Diagnostic Criteria on the Prevalence of Sarcopenia in a Large Middle Aged Cohort," Age, Vol. 35, No. 3, 2013, pp. 871-881. doi:10.1007/s11357-012-9384-Z

[3] I Janssen, S. B. Heymsfield, R. N. Baumgartner and R. Ross, "Estimation of Skeletal Muscle Mass by Bioelectrical Impedance Analysis," Journal of Applied Physiology, Vol. 89, No. 2, 2000, pp. 465-471.

[4] K. Sanada, C. F. Kearns, T. Midorikawa and T. Abe, "Prediction and Validation of Total and Regional Skeletal Muscle Mass by Ultrasound in Japanese Adults," European Journal of Applied Physiology, Vol. 96, No. 1, 2006, pp. 24-31. doi:10.1007/s00421-005-0061-0
[5] T. Midorikawa, K. Sanada, A. Yoshitomi and T. Abe, "Is the Use of Ultrasound-Derived Prediction Equations for Adults Useful for Estimating Total and Regional Skeletal Muscle Mass in Japanese Children?" British Journal of Nutrition, Vol. 101, No. 1, 2009, pp. 72-78. doi:10.1017/S000711450899440X

[6] J. Kim, Z. Wang, S. B. Heymsfield, R. N. Baumgartner and D. Gallagher, "Total-Body Skeletal Muscle Mass: Estimation by a New Dual-Energy X-Ray Absorptiometry Method," American Journal of Clinical Nutrition, Vol. 76, No. 2, 2002, pp. 378-383.

[7] J. Kim, W. Shen, D. Gallagher, A. Jones Jr, Z. Wang, J. Wang, S. Heshka and S. B. Heymsfield, "Total-Body Skeletal Muscle Mass: Estimation by Dual-Energy X-Ray Absorptiometry in Children and Adolescents," American Journal of Clinical Nutrition, Vol. 84, No. 5, 2006, pp. 1014-1020.

[8] T. Abe, M. Kondo, Y. Kawakami and T. Fukunaga, "Prediction Equations for Body Composition of Japanese Adults by B-Mode Ultrasound," American Journal of Human Biology, Vol. 6, No. 2, 1994, pp. 161-170. doi:10.1002/ajhb.1310060204

[9] T. Abe and T. Fukunaga, "Relationship between Subcutaneous Fat and Muscle Distributions and Serum HDLCholesterol," Journal of Atherosclerosis and Thrombosis, Vol. 1, No. 1, 1994, pp. 15-22.

[10] J. M. Bland and D. G. Altman, "Statistical Methods for Assessing Agreement between Two Methods of Clinical Measurement," Lancet, Vol. 8, No. 1, 1986, pp. 307-310. doi:10.1016/S0140-6736(86)90837-8

[11] T. Abe, C. F. Kearns and T. Fukunaga, "Sex Differences in Whole Body Skeletal Muscle Mass Measured by Magnetic Resonance Imaging and Its Distribution in Young Japanese Adults," British Journal of Sports Medicine, Vol. 37, No. 5, 2003, pp. 436-440. doi:10.1136/bjsm.37.5.436

[12] T. Abe, R. S. Thiebaud, J. P. Loenneke, M. G. Bemben, M. Loftin and T. Fukunaga, "Influence of Severe Sarcopenia in Cardiovascular Risk Factors in Nonobese Men," Metabolic Syndrome and Related Disorders, Vol. 10, No. 6, 2012, pp. 407-412. doi:10.1089/met.2012.0057

[13] T. W. Buford, D. J. Lott, E. Marzetti, S. E. Wohlgemuth, K. Vandenborne, M. Pahor, C. Leeuwenburgh and T. M. Manini, "Age-Related Differences in Lower Extremity Tissue Compartments and Associations with Physical Function in Older Adults," Experimental Gerontology, Vol. 47, No. 1, 2012, pp. 38-44. doi:10.1016/j.exger.2011.10.001 